\title{
Successful recanalization of complete bile duct obstruction using piercing technique under cholangioscopic guidance
}

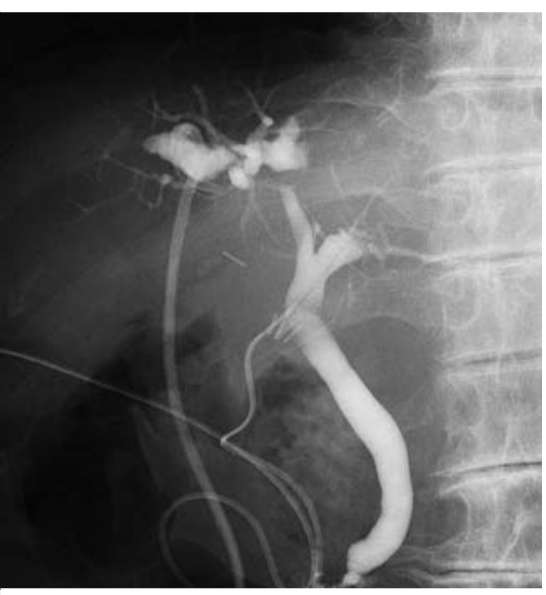

- Fig. 1 Simultaneous cholangiogram via the percutaneous drainage tube and the cystic duct tube placed into the common bile duct revealed complete shortsegment obstruction of the right hepatic duct.

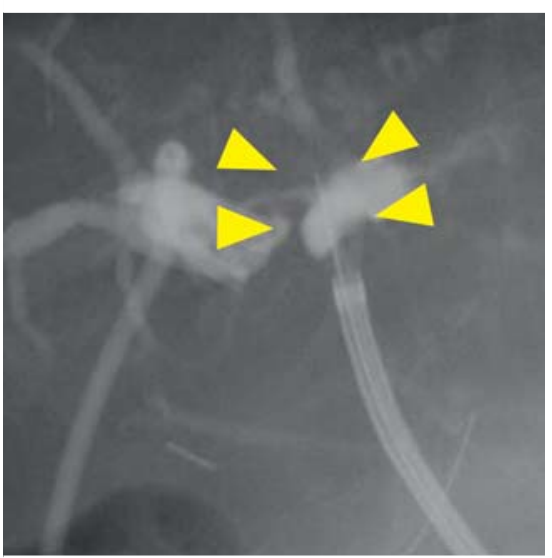

- Fig. 2 The cholangioscope tip was aligned with the direction of the right posterior branch, and piercing was performed with the stiff edge of the guidewire (yellow arrowheads).

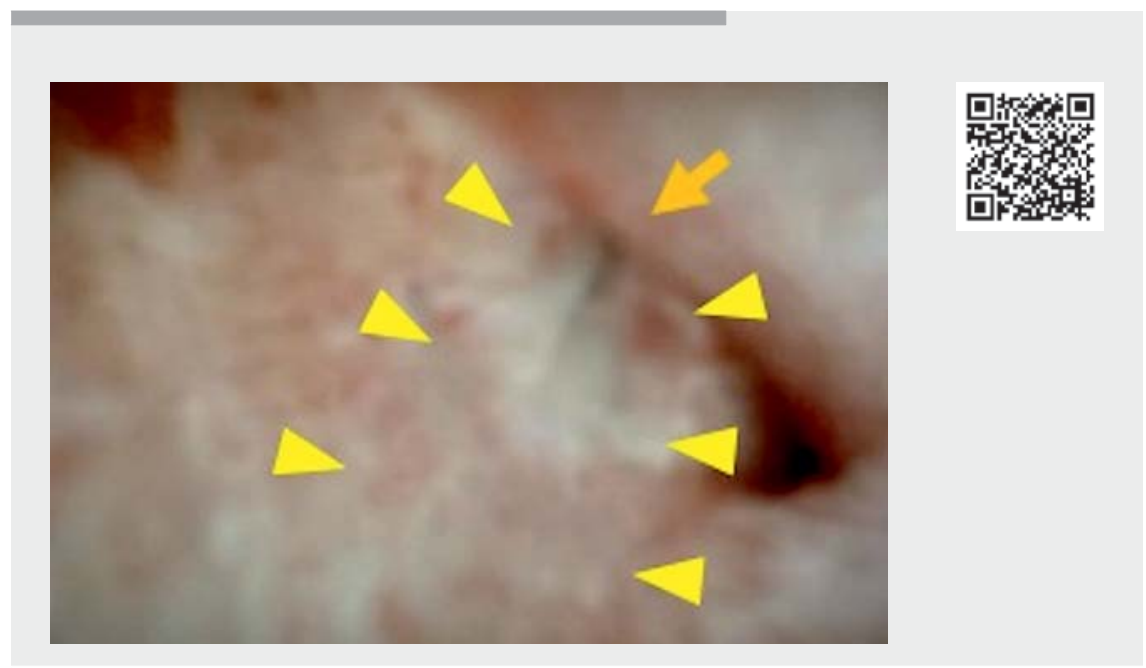

Video 1 Cholangioscopic imaging revealed the contrast medium flowing into the bile duct (yellow arrowheads) through a small hole created by the piercing (orange arrow). Finally, successful recanalization was achieved.

Bile duct strictures and obstructions after hepatectomy are significant complications. Endoscopic treatment is considered optimal because of its lesser invasiveness. However, complete obstruction is difficult to treat. We report the successful recanalization of complete bile duct obstruction using a piercing technique under cholangioscopic guidance.

A 79-year-old woman was referred to our department for the treatment of bile leakage with bile duct obstruction following right anterior sectionectomy. Endoscopic retrograde cholangiopancreatography $(E R C P)$ revealed obstruction of the right hepatic duct. Selective negotiation using a hydrophilic guidewire was not possible. Simultaneous cholangiogram via the percutaneous drainage tube and cystic duct tube placed into the common bile duct revealed complete shortsegment obstruction of the right hepatic duct (\$ Fig. 1). Contrast-enhanced computed tomography (CT) images showed no apparent intervening vessels between the right hepatic duct and the right posterior branch.
Selective negotiation under direct vision using a cholangioscope (SpyGlass DS; Boston Scientific, Natick, Massachusetts, USA) was attempted, but it was unsuccessful. Therefore, under fluoroscopic guidance, the cholangioscope tip was aligned with the direction of the right posterior branch, and piercing was performed with the stiff edge of the guidewire ( Fig. 2). Subsequently, negotiation of the right posterior branch was attempted, but the guidewire could not be advanced into the bile duct. However, cholangioscopic imaging revealed the contrast medium flowing into the bile duct through a small hole created by the piercing ( $\triangleright$ Video 1 ). Negotiation targeting this site under cholangioscopic guidance enabled selective guidewire insertion to the right posterior branch ( $\mathbf{F i g . 3}$ ). Then, the fistula was dilated using a 6-mm dilation balloon catheter (REN biliary dilation catheter; Kaneka, Osaka, Japan), and a 7-Fr plastic stent (Through \& Pass IS; Gadelius Medical, Tokyo, Japan) was placed across the fistula ( $\vee$ Fig.4). 

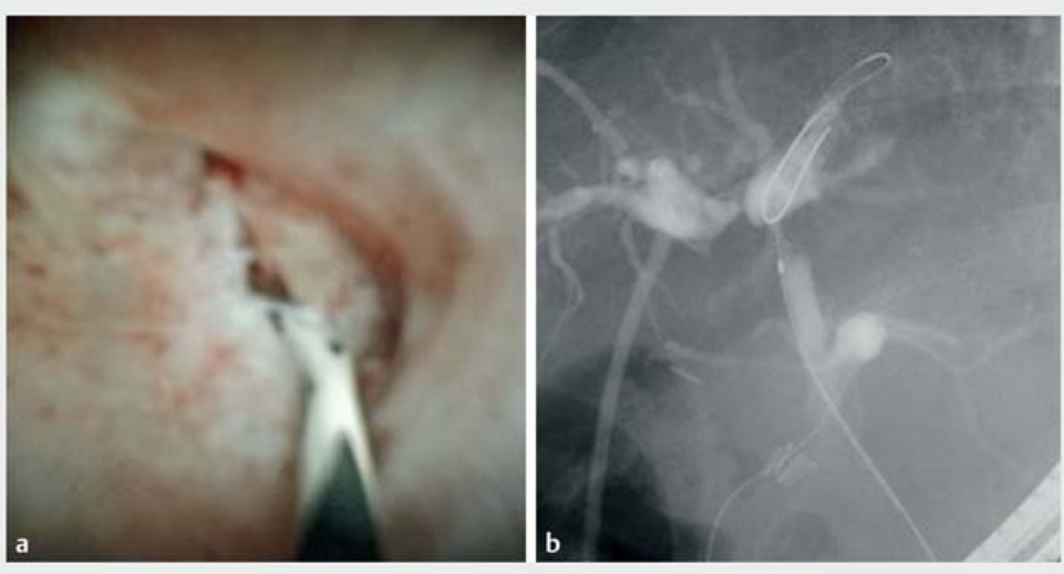

-Fig. 3 The cholangioscopic image revealed contrast medium flowing into the bile duct through a small hole created by the piercing. Negotiation targeting this site under cholangioscopic guidance enabled selective guidewire insertion to the right posterior branch. a Direct cholangioscopic image. b Fluoro-

Cholangioscopy-assisted guidewire placement and a piercing technique have been reported for postoperative biliary strictures or obstructions [1-5]. The combination of both as shown here is a useful alternative for difficult cases.

Endoscopy_UCTN_Code_CPL_1AK_2AD

\section{Competing interests}

The authors declare that they have no conflict of interest.

The authors

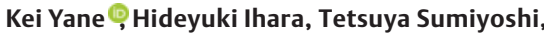
Mai Aoki, Masahiro Yoshida, Kotaro Morita, Hitoshi Kondo

Department of Gastroenterology, Tonan

Hospital, Sapporo, Hokkaido, Japan

Corresponding author

Kei Yane, MD

Department of Gastroenterology, Tonan

Hospital, 3-8 Kita-4 Nishi-7, Chuo-ku,

Sapporo 060-0004, Japan

Fax: +81-11-231-5000

k.yane3@gmail.com

\section{References}

[1] Martins FP, Ferrari AP. Cholangioscopyassisted guidewire placement in post-liver transplant anastomotic biliary stricture: efficient and potentially also cost-effective. Endoscopy 2017; 49: E283-E284

[2] Bukhari MA, Haito-Chavez Y, Ngamruengphong $S$ et al. Rendezvous biliary recanalization of complete biliary obstruction with direct peroral and percutaneous transhepatic cholangioscopy. Gastroenterology 2018; 154: 23-25

[3] Kim EH, Lee HG, Oh JS et al. Extraluminal recanalization of bile duct anastomosis obstruction after liver transplantation. J Vasc Interv Radiol 2018; 29: 1466-1471

[4] Rainer F, Blesl A, Spindelboeck W et al. A novel way to avoid reoperation for biliary strictures after liver transplantation: cholangioscopy-assisted guidewire placement. Endoscopy 2019; 51: E314-E316

[5] Toyonaga H, Hayashi T, Katanuma A. Piercing technique via cholangioscopy for the reconstruction of complete anastomotic obstruction after choledochojejunostomy. Dig Endosc 2020; 32: e86-e88

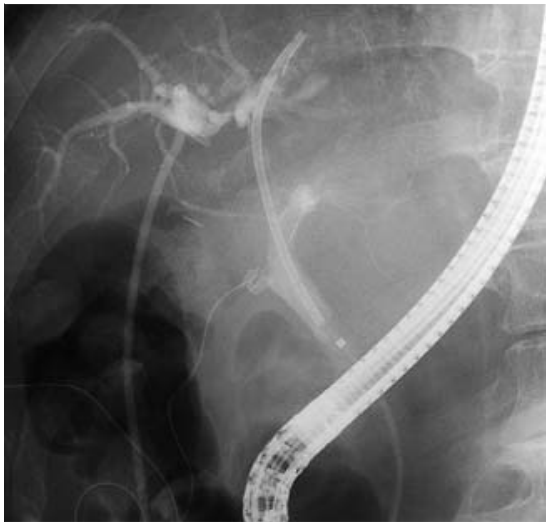

Fig. 4 A 7-Fr plastic stent was placed across the fistula.

Bibliography

Endoscopy 2022; 54: E348-E349

DOI 10.1055/a-1540-6735

ISSN 0013-726X

published online 19.7.2021

(c) 2021. Thieme. All rights reserved.

Georg Thieme Verlag KG, Rüdigerstraße 14,

70469 Stuttgart, Germany

\section{ENDOSCOPY E-VIDEOS}

https://eref.thieme.de/e-videos

口回 Endoscopy E-Videos is an open access online section, 靣保: reporting on interesting cases and new techniques in gastroenterological endoscopy. All papers include a high quality video and all contributions are freely accessible online. Processing charges apply (currently EUR 375), discounts and wavers acc. to HINARI are available.

This section has its own submission website at

https://mc.manuscriptcentral.com/e-videos 